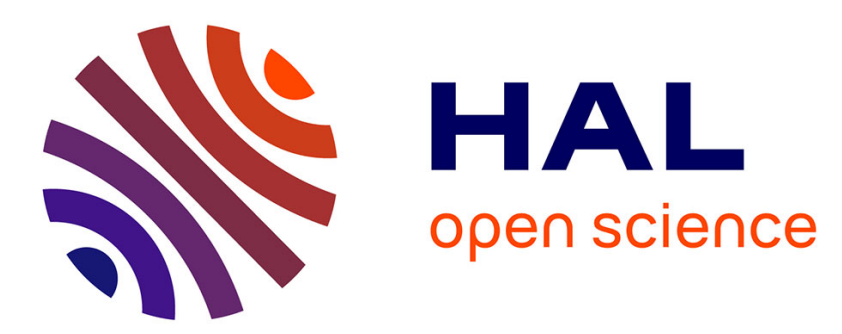

\title{
Molecular identification and stress response of the apoptosis-inducing factor gene 3 (AccAIF3) from Apis cerana cerana
}

Fang Wang, Yuanying Zhang, Pengbo Yao, Xingqi Guo, Han Li, Baohua Xu

\section{- To cite this version:}

Fang Wang, Yuanying Zhang, Pengbo Yao, Xingqi Guo, Han Li, et al.. Molecular identification and stress response of the apoptosis-inducing factor gene 3 (AccAIF3) from Apis cerana cerana . Apidologie, 2014, 45 (6), pp.685-700. 10.1007/s13592-014-0285-2 . hal-01234767

\section{HAL Id: hal-01234767 \\ https://hal.science/hal-01234767}

Submitted on 27 Nov 2015

HAL is a multi-disciplinary open access archive for the deposit and dissemination of scientific research documents, whether they are published or not. The documents may come from teaching and research institutions in France or abroad, or from public or private research centers.
L'archive ouverte pluridisciplinaire HAL, est destinée au dépôt et à la diffusion de documents scientifiques de niveau recherche, publiés ou non, émanant des établissements d'enseignement et de recherche français ou étrangers, des laboratoires publics ou privés. 


\title{
Molecular identification and stress response of the apoptosis-inducing factor gene 3 (Acc $A I F 3$ ) from Apis cerana cerana
}

\author{
Fang $\mathrm{WANG}^{1}$, Yuanying ZHANG ${ }^{1,3}$, Pengbo $\mathrm{YAO}^{1}$, Xingqi GuO ${ }^{1}$, $\mathrm{Han}_{\mathrm{LI}^{1}}$, Baohua $\mathrm{Xu}^{2}$ \\ ${ }^{1}$ State Key Laboratory of Crop Biology, College of Life Sciences, Shandong Agricultural University, Taian, \\ Shandong 271018, People's Republic of China \\ ${ }^{2}$ College of Animal Science and Technology, Shandong Agricultural University, Taian, Shandong 271018, People's \\ Republic of China \\ ${ }^{3}$ School of Basic Medical Sciences, Taishan Medical University, Taian, Shandong 271000, People's Republic \\ of China
}

Received 18 November 2013 - Revised 21 March 2014 - Accepted 2 April 2014

\begin{abstract}
Apoptosis-inducing factors (AIFs) play vital roles in bioenergetic and redox metabolism. Compared with studies focused on the apoptogenic function of AIF, few studies have reported on its oxidoreductase function, especially in insects. In this study, we identified a novel AIF gene AccAIF3 that was isolated from Apis cerana cerana, and investigated its expression and structural features. Quantitative real-time PCR (qRT-PCR) revealed the highest mRNA level of AccAIF3 in adult bees at 15 days. qRT-PCR results revealed that $A c c A I F 3$ transcripts were induced by cold, $\mathrm{CdCl}_{2}, \mathrm{HgCl}_{2}, \mathrm{UV}$, pyriproxyfen and cyhalothrin, but were downregulated by ecdysone. We also compared the expression of AIF3 from Apis cerana cerana and Apis mellifera ligustica Spinola to Ascosphaera apis, and found that AIF3s may respond to Ascosphaera apis in a species-specific manner. These data suggested that AccAIF3 may play vital roles in the response to abiotic and biotic stresses and contribute to the adaptability of honeybees to adversities.
\end{abstract}

\section{Apis cerana cerana / biotic and abiotic stresses / quantitative real-time PCR / AccAIF3}

\section{INTRODUCTION}

Apoptosis-inducing factor (AIF) is a phylogenetically conserved redox-active flavoprotein that is confined to mitochondria, where it exerts a vital function in bioenergetic and redox metabolism (Klein et al. 2002; Urbano et al. 2005). The first

Electronic supplementary material The online version of this article (doi:10.1007/s13592-014-0285-2) contains supplementary material, which is available to authorized users.

Corresponding author: H. Li, lihan@sdau.edu.cn;

B.Xu,bhxu@sdau.edu.cn

Manuscript editor: Klaus Hartfelder
AIF was identified and named by Guido Kroemer and colleagues (Susin et al. 1999; reviewed in Kroemer and Reed 2000). The crystal structures of mouse and human AIF revealed two important regions: a domain with homology to a bacterial NADH-dependent ferredoxin reductase and a domain required for DNA fragmentation (a putative DNA-binding site) (Maté et al. 2002; Ye et al. 2002). In most mammals, AIF also contains a mitochondrial localisation sequence (MLS) in its N-terminal (Lorenzo et al. 1999) and harbours two nuclear localisation signals (NLS) (Norberg et al. 2010). Two additional members are found in humans, apoptosis-inducing factor-like mitochondrion-associated inducer of death (AMID), which has also been found in the cytosol 
and has no MLS (Wu et al. 2002; Ohiro et al. 2002), and apoptosis-inducing factor-like (AIFL), which lacks a clear MLS (Xie et al. 2005). In addition to these homologues, some alternative splice forms of AIF are known: AIFexon2a, AIFexon2b, Ash, Ash2 and AIFsh3. The alternative usage of exon 2 (exon $2 \mathrm{a}$ or $2 \mathrm{~b}$ ) allows for the production of AIFexon2a (AIF1) and AIFexon2b (Loeffler et al. 2001; Otera et al. 2005). Ash is a short variant that contains NLS and the proapoptotic segment, but lacks the MLS and redoxactive domain (Delettre et al. 2006a). AIFsh2 localises to mitochondria and has redox function, but lacks the pro-apoptotic domain. AIFsh3 resembles AIFsh2, although it lacks MLS (Delettre et al. 2006b). Among all the forms, AIF is most abundant, so we chose AIF as our experimental subject. As its name indicates, AIF may function in the life of the cell.

Confronted with environmental stresses, in principle, cells can react in two distinct ways. To repair damage or resume normal cellular functions, some of the stressed cells can activate defence mechanisms to adapt to stressful conditions. On the other hand, programmed cell death can be activated, leading to apoptosis. The deciding factors in the choice between these two pathways are the stress intensity and cellintrinsic parameters (Garrido et al. 2003; Mosser and Morimoto 2004; Thompson 1995). Previous studies have identified that mitochondria have a dual role in cells. Mitochondria generate ATP by oxidative phosphorylation and play a central role in metabolic pathways. In addition, mitochondria play an important role in the regulation of cell death (Kroemer 1997; Green and Reed 1998). As a mitochondrial protein, AIF also functions to activate these two opposing ways.

Initial studies concerning AIFs were focused on its caspase-independent apoptotic function. AIF appears to be a caspase-independent death factor (Susin et al. 1999). $\mathrm{AIF}^{-/ Y} \mathrm{ES}$ cells are sensitive to various apoptotic stimuli, such as ultraviolet irradiation (UV) and anisomycin (Joza et al. 2001). AIF is essential for the first wave of cell death during mouse morphogenesis. As discussed previously, the addition of recombinant AIF to purified nuclei causes chromatin condensation (Susin et al. 1999).

However, recent in vivo data indicate that in addition to its lethal activity, AIF plays a vital role in the mitochondria of healthy cells by regulating the activity of the mitochondrial respiratory chain complex I; this form of AIF is dispensable for apoptosis and has an NADH oxidase domain (Hangen et al. 2009, 2010). Other studies have shown that AIF has lifesupporting mitochondrial activity (Joza et al. 2001; Miramar et al. 2001). AIF has been proposed to act as a putative reactive oxygen species (ROS) scavenger, but this hypothesis remains to be confirmed (Klein et al. 2002). Another subsequent work showed that the loss of AIF leads to an increase in ROS, and exogenous antioxidants can alleviate the complex I deficiency caused by AIF depletion (Apostolova et al. 2006). Compared with studies in mammals, few studies have focused on insects. D. melanogaster AIF (DmAIF) is highly conserved in the regions of mouse AIF $(m A I F)$ which was critical for its oxidoreductase activity (Joza et al. 2008).

Chinese honeybees (Apis cerana cerana) play a vital role in the balance of native ecologies and economic crops. Compared with Apis mellifera ligustica Spinola (A. mellifera L.), A. cerana cerana has unique characteristics, such as heat hardiness and disease resistance, as well as the pollination of flowering crops at low temperature ( $\mathrm{Li}$ et al. 2008). Environmental pollution threatens the existence of $A$. cerana cerana. In order to protect $A$. cerana cerana, we chose it as experimental insect to investigate its defences against oxidant stresses. In this study, we cloned and characterised AIF3 from $A$. cerana cerana and detected its levels of expression at various developmental stages. We also cloned the $5^{\prime}$ flanking region of AccAIF3 and predicted some putative cisacting elements. qRT-PCR results revealed that the AccAIF3 transcript was upregulated in response to various environmental stresses. Our study may contribute to the knowledge of AIF in insects and protect Chinese honeybees from injury. 


\section{MATERIALS AND METHODS}

\subsection{Biological specimens and treatment}

A. cerana cerana and A. mellifera $\mathrm{L}$. were maintained at the experimental apiary of Shandong Agricultural University, Taian, China. Eggs were collected from the honeycomb. Using age, eye colour and shape, we divided worker honeybees into ten groups: larvae from the first to fifth instars (L1-L5), prepupae (PP) and pupae with white eyes (Pw), pink eyes $(\mathrm{Pp})$, brown eyes $(\mathrm{Pb})$ and dark eyes $(\mathrm{Pd})$ (Michelette and Soares 1993). We labeled bees to distinguish their ages. The one-day-old adult worker honeybees (A1) were collected soon after they emerged from the honeycomb, while 15-day postemergence (A15) and 30-day post-emergence (A30) adults were collected at the entrance of the hive (Yan et al. 2012). The number of collected A30 groups was 10, and the number of collected A15 groups was 300 to do experiments. Worker bees collected were maintained in wooden cages $(20 \times 15 \times 10 \mathrm{~cm})$ covered with transparent plastic, and the number of bees per cage was 50. The adults were fed basic adult food containing $30 \%$ honey from the source colonies, $70 \%$ powdered sugar and water (Alaux et al. 2010) in an incubator with $60 \%$ relative humidity at $34{ }^{\circ} \mathrm{C}$ under a $24-\mathrm{h}$ dark regimen. A15 adults were divided into nine groups $(n=25)$. Groups $1-3$ were treated at $4{ }^{\circ} \mathrm{C}, 25{ }^{\circ} \mathrm{C}$ and $42{ }^{\circ} \mathrm{C}$. The controls for groups $1-3$ were incubated at $34^{\circ} \mathrm{C}$. Groups $4-6$ were treated with pesticides (pyriproxyfen, cyhalothrin and phoxime) as food. The pesticides were dissolved in food at a final concentration of $20 \mathrm{mg} / \mathrm{L}$ and $0.5 \mu \mathrm{L}$ were delivered to each bee in the thoracic notum. All insecticides were purchased from Huayang Technology Co., Ltd. (Shandong, China). The controls for groups 4-6 were fed normal food. Group 7 was subjected to ultraviolet (UV) light $\left(30 \mathrm{mj} / \mathrm{cm}^{2}\right)$. Groups 8 and 9 were fed a pollen and sucrose solution containing $\mathrm{HgCl}_{2}$ ( $3 \mathrm{mg} / \mathrm{mL})$ and $\mathrm{CdCl}_{2}(3 \mathrm{mg} / \mathrm{mL})$, and each bee was fed a mixture containing pollen and $\mathrm{HgCl}_{2}$ or $\mathrm{CdCl}_{2}$ $(3 \mu \mathrm{g})$ with a micropipette. The controls for groups 8 and 9 were fed the basic adult diet. For ecdysone treatment, L3 were placed in 24-well plates and fed food dissolved in a pollen and sucrose solution at a final concentration of $1.0 \mu \mathrm{g} / \mathrm{mL}$ and $0.001 \mu \mathrm{g} / \mathrm{mL}$. $A$. cerana cerana L3 and $A$. mellifera $\mathrm{L}$. L3 were placed in 24-well plates and fed brood food containing Ascosphaera apis (spore numbers to $10^{6} / \mathrm{mL}$ ). The L3 control group was fed normal food. Three honeybees were harvested at the appropriate time (Table I) for each condition and were stored at $-80{ }^{\circ} \mathrm{C}$ until they were analysed. Each treatment had three replicates.

\subsection{Primers}

Primers used for PCR amplification are listed in Table II.

\subsection{Extraction of total RNA, isolation of genomic DNA and cDNA synthesis}

Total RNA was extracted from worker honeybees using Trizol reagent (Invitrogen, Carlsbad, CA) and
Table I. The collection time (post treatment) for each experimental group

\begin{tabular}{ll}
\hline Experiment condition & Collection time after treatment \\
\hline $4{ }^{\circ} \mathrm{C}$ & $0.5,1,1.5,2$, and $2.5 \mathrm{~h}$ \\
$25{ }^{\circ} \mathrm{C}$ & $0.5,1.5,2.5,3.5$, and $4.5 \mathrm{~h}$ \\
$42{ }^{\circ} \mathrm{C}$ & $0.5,1,1.5,2$, and $2.5 \mathrm{~h}$ \\
pyriproxyfen & $1,2,3,4,5$ and $6 \mathrm{~h}$ \\
cyhalothrin & $1,2,3$, and $4 \mathrm{~h}$ \\
phoxime & $0.5,1,1.5,2$ and $2.5 \mathrm{~h}$ \\
$\mathrm{UV}$ & $1,2,3,4$ and $5 \mathrm{~h}$ \\
$\mathrm{HgCl}_{2}$ & $3,6,9$ and $12 \mathrm{~h}$ \\
$\mathrm{CdCl}_{2}$ & $3,6,9$ and $12 \mathrm{~h}$ \\
ecdysone & $3,6,9$ and $12 \mathrm{~h}$ \\
Ascosphaera apis & 1,2 and $3 \mathrm{~d}$ \\
\hline
\end{tabular}


Table II. The primers used in this study

\begin{tabular}{|c|c|c|}
\hline Abbreviation & Primer sequence( $\left(5^{\prime}-3^{\prime}\right)$ & Description \\
\hline PU & CCACATGCAATTCGAGGTCAATC & Internal fragment primer, forward \\
\hline PD & GGATCTGCCGCAACACTACTCAT & Internal fragment primer, reverse \\
\hline $5 \mathrm{P} 1$ & GCTTTCACGCGTACAAAACCAGATG & 5' RACE reverse primer, primary \\
\hline $5 \mathrm{P} 2$ & CCTGTGTGAAGTAAAGCCCCAT & 5' RACE reverse primer, nested \\
\hline AAP & GGCCACGCGTCGACTAGTAC $(\mathrm{G})_{14}$ & Abridged anchor primer \\
\hline AUAP & GGCCACGCGTCGACTAGTAC & Abridged universal amplification primer \\
\hline $3 \mathrm{P} 1$ & GCAGTCTCCTATCGAAATGCTACC & 3' RACE reverse primer, primary \\
\hline 3P2 & TTTCAGCTGCAATAGGTCATTATCC & 3' RACE reverse primer, nested \\
\hline B25 & GACTCGAGTCGACATCGAT & Universal primer, nested \\
\hline B26 & GACTCGAGTCGACATCGA $(\mathrm{T})_{18}$ & Universal primer, primary \\
\hline QCU & CCACATGCAATTCGAGGTCAATC & Full length primer, forward \\
\hline QCD & GCAATTTCATTCAATTTTCCACTTG & Full length primer, reverse \\
\hline N1 & GGGCGGAAAAAATTGTAAAGGACT & Genomic sequence primer, forward \\
\hline N2 & GCTTTCACGCGTACAAAACCAGAT & Genomic sequence primer, reverse \\
\hline N3 & CATCTGGTTTTGTACGCGTGAAAG & Genomic sequence primer, forward \\
\hline N4 & GGATCTGCTGCAACACTACTCATAGC & Genomic sequence primer, reverse \\
\hline RTU & TGGGGCTTTACTTCACACAGG & qRT-PCR perimer, forward \\
\hline RTD & GCTTTCACGCGTACAAAACCAGATG & qRT-PCR perimer, reverse \\
\hline QDU & GCTGGACCTTCTTGTGTGAT & 5' flanking region primer, forward \\
\hline QDD & GCCTGGTAGAGTATTAGAATTTGGT & $5^{\prime}$ flanking region primer, nested \\
\hline RTU & TGGGGCTTTACTTCACACAGG & qRT-PCR perimer, forward \\
\hline RTD & GCTTTCACGCGTACAAAACCAGATG & qRT-PCR perimer, reverse \\
\hline$A R P 1-s$ & GTTTTCCCATCTATCGTCGG & Standard control primer, forward \\
\hline$A R P 1-x$ & TTTTCTCCATATCATCCCAG & Standard control primer, reverse \\
\hline$A R P 1-s-y$ & TTGTATGCCAACACTGTCCTTT & A. mellifera standard control primer, forward \\
\hline$A R P 1-x-y$ & AGAATTGACCCACCAATCCA & A. mellifer $a$ standard control primer, reverse \\
\hline RTU-y & TGGGGCTTTACTTCATACAGG & A. mellifera AIF3, qRT-PCR primer, forward \\
\hline RTD-y & CGCTTTCACGTGTACAAAACCAGATG & A. mellifera AIF3, qRT-PCR primer, reverse \\
\hline
\end{tabular}

digested with RNase-free DNaseI (Promega, Madison, WI). The first stand cDNA was synthesised using a reverse transcriptase system (TransGen Biotech, Beijing, China). Genomic DNA was isolated from worker bees using the EasyPure Genomic DNA Extraction Kit (TransGen Biotech, Beijing, China).

\subsection{Full-length cDNA isolation of $A c c A I F 3$}

The full-length cDNA of AccAIF3 was isolated by reverse transcription-PCR (RT-PCR) and rapid amplification of cDNA ends-PCR (RACE-PCR).
Primers PU and PD were designed to amplify the internal fragment, and primers $5 \mathrm{P} 1 / 5 \mathrm{P} 2,3 \mathrm{P} 1 / 3 \mathrm{P} 2$, AAP/AUAP and B26/B25 were designed for $5^{\prime}$ RACE and 3' RACE (all primers are listed in Table II). Finally, the full-length cDNA of AccAIF3 was obtained using primers QCU and QCD (primers are listed in Table II). All products were purified, cloned into the pEASY-T3 vector (TransGen Biotech, China), transformed into the Escherichia coli strain DH5 $\alpha$ and then sequenced. All sequencing was done by BioSune using ABI $3730 \mathrm{XL}$ sequenator. 


\subsection{Amplification of the 5 ' flanking region and amplification of the genomic sequence of $A c c A I F 3$}

The promoter region was amplified by the inverse polymerase chain reaction (I-PCR) method using specific primers (QD51/QD31 and QD52/QD32) (primers are listed in Table II). The template was genomic DNA digested by restriction endonuclease EcoRI and then self-ligated by T4 DNA ligase (TakaRa, Dalian, China). Primers QDU and QDD (primers are listed in Table II) were used to obtain the full-length sequence of 5' flanking region. Transcription factor binding sites (TFBS) were searched in the 5 ' flanking region using the MatInspector database (http:// www.cbrc.jp/research/db/TFSEARCH.html) and TRANSFAC database (http://www.generegulation.com/pub/databases.html). To amplify the genomic sequence of AccAIF3, two pairs of specific primers (N1/N2 and N3/N4; shown in Table II) were designed and used. All PCR products were purified, cloned into the pEASY-T3 vector (TransGen Biotech, China) and transformed into the Escherichia coli strain $\mathrm{DH} 5 \alpha$ for sequencing. All sequencing was done by BioSune using ABI 3730 XL sequenator.

\subsection{Bioinformatic analysis}

Conserved domains of AccAIF3 were analysed and compared using the BLAST algorithm available at NCBI server (http://blast.ncbi.nim.nih.gov/Blast.cgi and http://www.ncbi.nlm.nih.gov/Structure/cdd/ wrpsb.cgi?RID=54139JC601 R\&mode=all). Using DNAMAN software 5.2.2 developed by the American Lynnon Biosoft Company (USA), we identified the open reading frame (ORF) and conducted multiple alignments amongst homologues. A phylogenetic tree was generated using Molecular Evolutionary Genetic Analysis (MEGA version 4.0). The web software program TFSEARCH (http://www.cbrc.jp/research/db/ TFSEARCH.html) was used to predict the cis-elements in the promoter region.

\subsection{The transcription expression level of AccAIF3 using quantitative PCR}

Total RNA was extracted from samples from different treatments, and the first strand cDNA was synthesised as described above. qRT-PCR was performed with a modified qRT-PCR volume containing $1.6 \mu \mathrm{L}$ of diluted cDNA from different samples, $10.0 \mu \mathrm{L}$ of Takara SYBR ${ }^{\circledR}$ premix Ex Taq ${ }^{\mathrm{TM}}, 0.4 \mu \mathrm{L}$ of each primer $(10 \mathrm{pmol} / \mathrm{mL})$, and $7.6 \mu \mathrm{L}$ of $\mathrm{ddH} 2 \mathrm{O}$ from Yao et al. 2013 using primers RTU/RTD designed based on the sequences of AccAIF3, primer ARP1-S/ $A R P 1-x$ designed based on the A. cerana cerana ARP1 gene (GenBank accession no. HM640276) and primers ARP1-s-y/ARP1-x-y designed based on the A. mellifera (GenBank accession no. GB44311). ARP1 was selected for the normalization of RNA levels (Yao et al. 2014), and we also tested the expression stability of this endogenous control gene (date not show). Primers RTU-y/ RTd-y were designed based on the A. mellifera AIF3 gene (GenBank accession NM_001185146.1). All primers above are listed in Table II. The relative AccAIF3 gene expression was analysed by the comparative CT method $\left(2^{-\Delta \Delta C T}\right.$ method). At least three individual samples were prepared for each sample and all samples were analysed in three replicates.

\subsection{Statistical analysis}

Error bars denote the standard error of the mean (SEM) from three independent experiments. Significant differences were determined by Duncan's multiple range tests using the Statistical Analysis System (SAS) version 9.1 software programs (SAS Institute, Cary, NC, USA).

\section{RESULTS}

\subsection{Isolation and sequence analysis of the full-length cDNA of AccAIF3}

We cloned the cDNA sequence of $A I F 3$ from $A$. cerana cerana (GenBank accession number: KF745895). Sequence analysis indicates that the cDNA is 2586 bp long, containing a 1734 bp ORF, a 622 bp 5 ' untranslated region (UTR) and a 230 bp 3' UTR. The ORF encodes a protein of 577 amino acids. Figure 1a shows that AccAIF3 is most similar to A. mellifera AIF3 (AmAIF3, $96.37 \%$ identity, GenBank ID: XP_625035.2).

The conserved domains of AccAIF3 were identified by NCBI Conserved Domain Database Search, and included a Rieske domain 
a
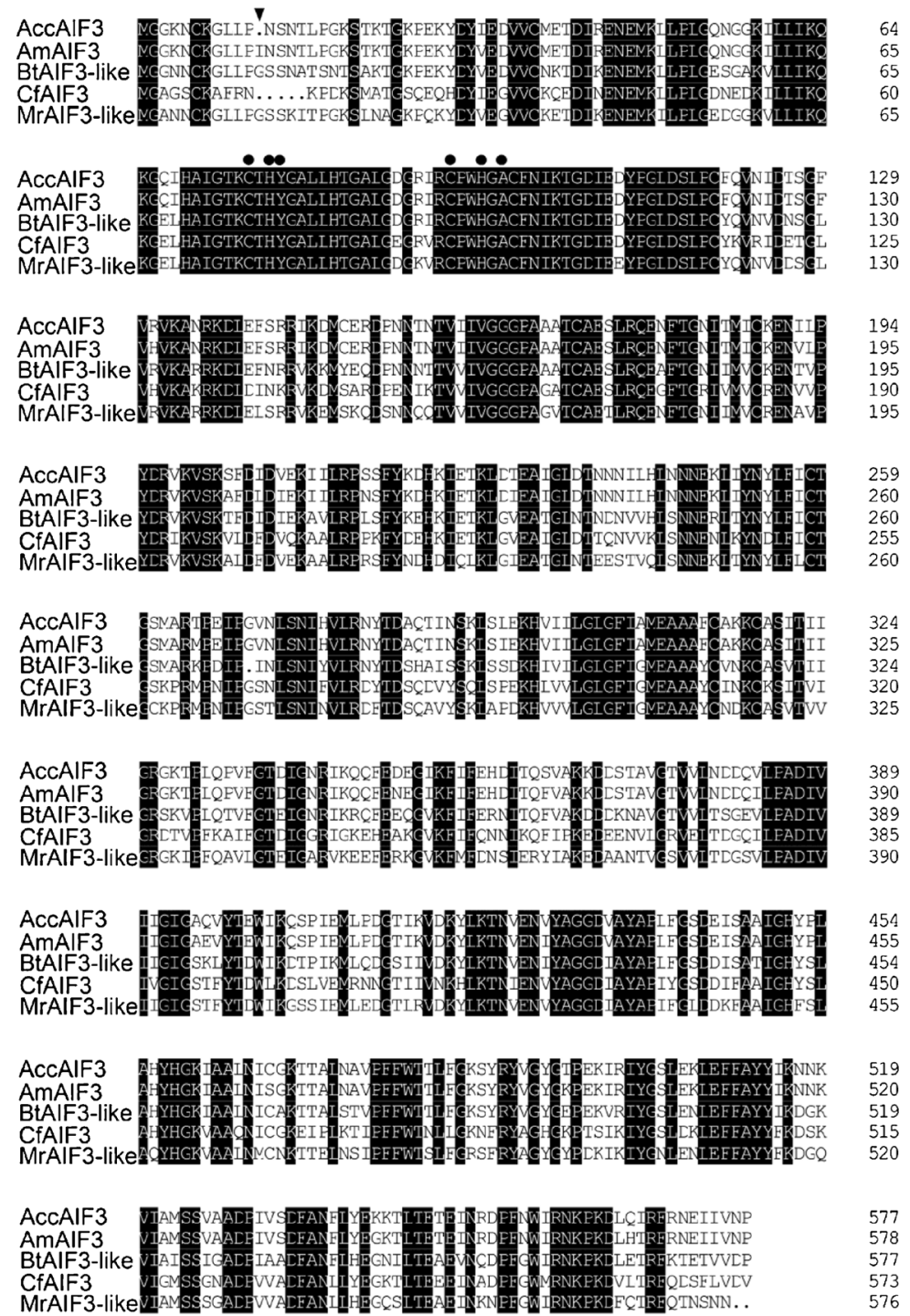

b

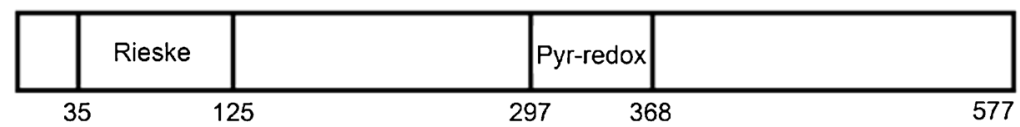

Figure 1 a Characterisation of apoptosis-inducing factors (AIFs) from various species. An alignment of the identified AccAIF3 protein sequence (GenBank ID: KF745895) with other known insect AIF3s. The amino acid sequences used in the analysis were from A. mellifera (AmAIF3, GenBank ID: XP_625035.2), Bombus terrestris (BtAIF3-like, XP_003695310.1), Camponotus floridanus (Cfaif3, EFN71795.1) and Megachile rotundata (Mraif3-like, XP_003703654.1). Identical amino acid residues are shaded in black. A triangle was used to show the specific amino acid that exists in A. mellifera but does not exist in A. cerana cerana. Circles were used to show the $[2 \mathrm{Fe}-2 \mathrm{~S}]$ cluster binding site. $\mathbf{b}$ The conserved domains of AccAIF3 
and a pyridine nucleotide-disulfide oxidoreductase domain (Pyr_redox) (Fig. 1b). The Rieske domain, a [2Fe-2S] cluster binding domain, is involved in electron transfer. The Pyr_redox domain is a small NADH binding domain within a larger FAD binding domain. A phylogenetic tree of AIFs from different species was constructed using a neighbour-joining method implemented in MEGA, and showed the possible evolutionary relationship among AIFs (Fig. 2). Phylogenetic protein sequence analysis revealed that AccAIF3 was more closely related to AmAIF3, respectively, than other homologues. In addition, this result was in agreement with the relationship predicted from the multiple sequence alignment.

\subsection{The genetic structure of $A c c A I F 3$}

The full-length DNA sequence of AccAIF3 is 4051 bp long, containing six introns inserted within seven exons (GenBank accession number: KF745896). All introns have classical characteristics: they are flanked by the 5'-GT splice donor and 3'-AG splice donor and exhibit high AT content. To further investigate the features of AccAIF3, a comparison was performed. According to Table III and Figure 3, the results indicated that the AT content of the introns was really much higher than that in exons. In addition, the length of exons or introns in $A I F 3 s$ from different species was also highly conserved; especially in $A$. cerana.

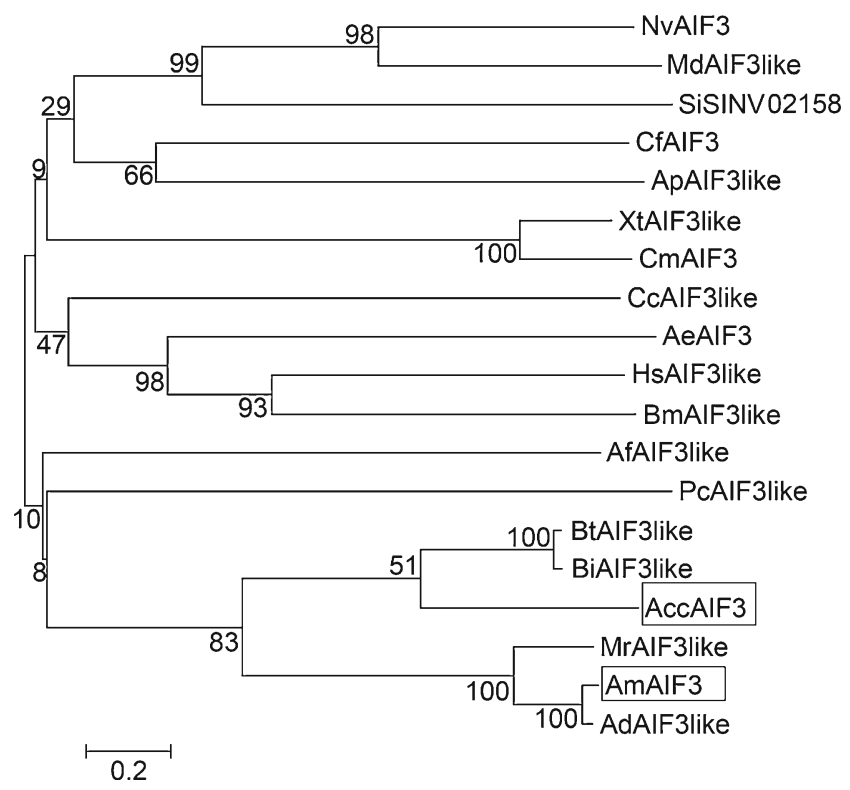

Figure 2 A phylogenetic tree of AIF3s was constructed from different species using the neighbour-joining method and MEGA 4.0 software. The amino acid sequences of the AIF3s obtained from NCBI are as follows: NvAIF3 (Nasonia vitripennis, XP_001607016.1), MdAIF3-like (Musca domestica, XP 005183902.1), Si SINV_02158 (Solenopsis invicta, EFZ18689.1), Cfaif3 (Camponotus floridanus, EFN71795.1), ApAIF3-like (Acyrthosiphon pisum, XP_003241906.1), XtAIF3-like (Xenopus (Silurana) tropicalis, XP_002936104.1), CmSIF3 (Chelonia mydas, EMP37830.1), CcAIF3-like (Ceratitis capitata, XP_004518444.1), AeAIF3 (Acromyrmex echinatior, EGI65010.1), HsAIF3 (Harpegnathos saltator, EFN̄79971.1), BmAIF3-like (Bombyx mori, XP_004926894.1), AfAIF3-like (Apis florea, XP_003695310.1), PcAIF3-like (Pomacea canaliculata, AFQ23946.1), BtAIF3-like (Bombus terrestris, XP_003695310.1), BiAIF3-like (Bombus impatiens, XP_003489952.1), Mraif3-like (Megachile rotundata, XP_003703654.1), AmAIF3 (A. mellifera, GenBank ID: XP_625035.2) and AdAIF3-like (Apis dorsata, XP_006620108.1) 
Table III. The lengths of the exons and introns and the AT content in AccAIF3, AmAIF3 and MrAIF3-like

\begin{tabular}{|c|c|c|c|c|c|c|c|}
\hline Exon & Gene & $\begin{array}{l}\text { Length } \\
\text { (bp) }\end{array}$ & $\begin{array}{l}\mathrm{AC} \text { content } \\
(\%)\end{array}$ & Intron & Gene & $\begin{array}{l}\text { Length } \\
\text { (bp) }\end{array}$ & $\begin{array}{l}\text { AT content } \\
(\%)\end{array}$ \\
\hline 1 & $\begin{array}{l}\text { AccAIF3 } \\
\text { AmAIF3 } \\
\text { MrAIF3-like }\end{array}$ & $\begin{array}{l}70 \\
73 \\
73\end{array}$ & $\begin{array}{l}63 \\
64 \\
54\end{array}$ & 1 & $\begin{array}{l}\text { AccAIF3 } \\
\text { AmAIF3 } \\
\text { MrAIF3-like }\end{array}$ & $\begin{array}{l}1062 \\
1153 \\
1012\end{array}$ & $\begin{array}{l}84 \\
84 \\
72\end{array}$ \\
\hline 2 & $\begin{array}{l}\text { AccAIF3 } \\
\text { AmAIF3 } \\
\text { MrAIF3-like }\end{array}$ & $\begin{array}{l}292 \\
293 \\
293\end{array}$ & $\begin{array}{l}66 \\
66 \\
64\end{array}$ & 2 & $\begin{array}{l}\text { AccAIF3 } \\
\text { AmAIF3 } \\
\text { MrAIF3-like }\end{array}$ & $\begin{array}{l}61 \\
62 \\
70\end{array}$ & $\begin{array}{l}87 \\
89 \\
79\end{array}$ \\
\hline 3 & $\begin{array}{l}\text { AccAIF3 } \\
\text { AmAIF3 } \\
\text { MrAIF3-like }\end{array}$ & $\begin{array}{l}337 \\
337 \\
337\end{array}$ & $\begin{array}{l}69 \\
68 \\
61\end{array}$ & 3 & $\begin{array}{l}\text { AccAIF3 } \\
\text { AmAIF3 } \\
\text { MrAIF3-like }\end{array}$ & $\begin{array}{l}66 \\
65 \\
59\end{array}$ & $\begin{array}{l}90 \\
93 \\
75\end{array}$ \\
\hline 4 & $\begin{array}{l}\text { AccAIF3 } \\
\text { AmAIF3 } \\
\text { MrAIF3-like }\end{array}$ & $\begin{array}{l}351 \\
351 \\
351\end{array}$ & $\begin{array}{l}73 \\
74 \\
64\end{array}$ & 4 & $\begin{array}{l}\text { AccAIF3 } \\
\text { AmAIF3 } \\
\text { MrAIF3-like }\end{array}$ & $\begin{array}{l}87 \\
106 \\
69\end{array}$ & $\begin{array}{l}90 \\
90 \\
76\end{array}$ \\
\hline 5 & $\begin{array}{l}\text { AccAIF3 } \\
\text { AmAIF3 } \\
\text { MrAIF3-like }\end{array}$ & $\begin{array}{l}206 \\
206 \\
206\end{array}$ & $\begin{array}{l}68 \\
68 \\
61\end{array}$ & 5 & $\begin{array}{l}\text { AccAIF3 } \\
\text { AmAIF3 } \\
\text { MrAIF3-like }\end{array}$ & $\begin{array}{l}99 \\
96 \\
61\end{array}$ & $\begin{array}{l}87 \\
85 \\
76\end{array}$ \\
\hline 6 & $\begin{array}{l}\text { AccAIF3 } \\
\text { AmAIF3 } \\
\text { MrAIF3-like }\end{array}$ & $\begin{array}{l}217 \\
217 \\
217\end{array}$ & $\begin{array}{l}69 \\
69 \\
64\end{array}$ & 6 & $\begin{array}{l}\text { AccAIF3 } \\
\text { AmAIF3 } \\
\text { MrAIF3-like }\end{array}$ & $\begin{array}{l}90 \\
100 \\
69\end{array}$ & $\begin{array}{l}88 \\
88 \\
76\end{array}$ \\
\hline 7 & $\begin{array}{l}\text { AccAIF3 } \\
\text { AmAIF3 } \\
\text { MrAIF3-like }\end{array}$ & $\begin{array}{l}260 \\
260 \\
254\end{array}$ & $\begin{array}{l}72 \\
73 \\
63\end{array}$ & & & & \\
\hline
\end{tabular}

cerana and $A$. mellifera, which agrees with the relationship predicted from the multiple sequence alignment.

\subsection{Characterisation of the $5^{\prime}$ flanking region of $A c c A I F 3$}

To investigate the regulatory region of AccAIF3, a $663 \mathrm{bp}$ fragment upstream of the transcription start site was cloned by I-PCR. Some putative TFBS were predicted and are shown in Figure 4. In addition, the basic TATA-box and CAAT-box, which are critical for transcription, were also found in the 5' flanking region (data not shown). All the TFBS indicated that AcCAIF3 might be involved in the regulation of stress and development at the level of transcription.

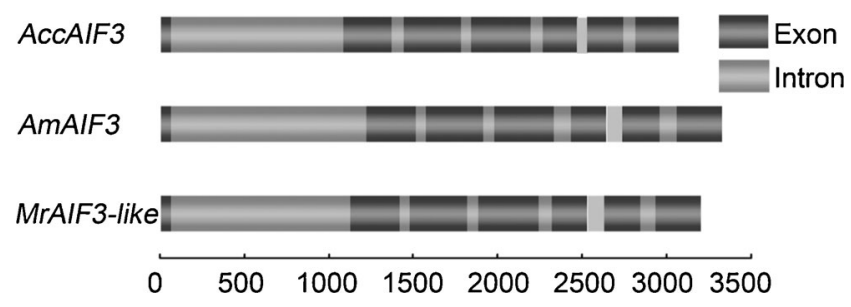

Figure 3 Comparison of Genomic structures among the $A I F 3 s$ gene family. Lengths of genomic DNA sequences of AccAIF3 (KF745896) and its homologues, including AmAIF3 (NC_007076.3) and MrAIF3-like (NW_003797177.1), are shown. The exons are indicated with black boxes, and the introns are indicated with grey boxes 


\begin{tabular}{|c|c|}
\hline TGATGAAATTTTTTTACTTTTTAAGTAATTACAATATATTATACATCAATTTTC & -610 \\
\hline BR-C, SRK HSF & \\
\hline ATTTAAA AATTATATTTCAATTTCATAT TTCATAAAGAAAAAATTTTAATTAAT & -556 \\
\hline AATCTCGATAAATATTAAATAATTTAATTTATAATTTGTATTATTAATA AATTTAT & -501 \\
\hline $\begin{array}{c}\text { BR-C } \\
\text { CATATAATAACAGATATATAAAACACAATGTTAAAACTTTCTAAAC }\end{array}$ & -449 \\
\hline ATTTTAGCCA ATTCAAATTAATTCAAATCAAATATTATAAAATGCTCTTTCTT & -396 \\
\hline $\begin{array}{c}\text { CF-\| CF- } \| \\
\text { CTTTCATATATATATACAAACATGAAATATTTTGTGTATACAATTCACATCTT }\end{array}$ & -342 \\
\hline $\begin{array}{ccc}\text { Oct-1 } & \text { SKY } \quad \text { HSF } & \text { NIT2 } \\
\text { TATTCATAATGAAA AAAGAAA GGCAATCTCAACATAAAAGTATATCAAATAT }\end{array}$ & -290 \\
\hline CdxA & \\
\hline TA AGAAT GATAAATATTTTATTGCATTAACAAATTTTTTTTTTAATATAAT & -236 \\
\hline $\mathrm{CdXA}$ & \\
\hline $\begin{array}{l}\text { AATAAAATTAATATGCAATATAAAAT TTAATAATGTTATAAACA AGAATATTC } \\
\text { Oct- } 1\end{array}$ & -183 \\
\hline ATAAAAATATGAGATTTCATACTGAATTGTAAGAGAGCGCTGATGGATACAA & -131 \\
\hline AATAAACTTACATCATTTCTTGTTCATGTTTCTAATCTATATTATTTTATTTGTT & -76 \\
\hline CF-II & \\
\hline AATGCTATATCATATATATATATATATATATATATACATAACATCGTATGCGCAC & -21 \\
\hline GAAAATCGATTTCCTTAAAAG & +10 \\
\hline
\end{tabular}

Figure 4 Characterisation of the $5^{\prime}$ flanking region in AccAIF3. The transcription start site is marked with an arrowhead. The predicted transcription factor binding sites mentioned in the text are marked as follows: HSF, CF2-II, Dfd, SKY, SRK, Oct-1, NIT2 and BR-C

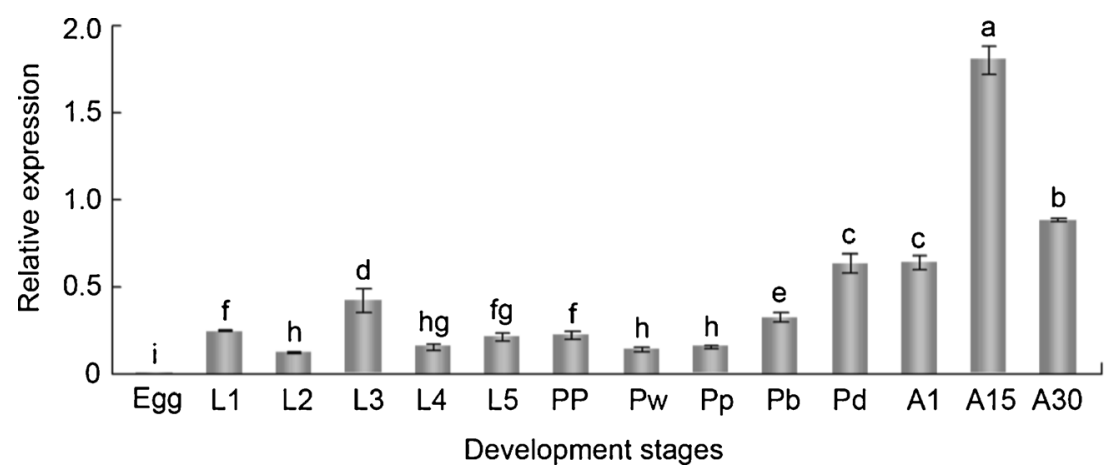

Figure 5 The expression patterns of AccAIF3. mRNA expression of AccAIF3 at different developmental stages: eggs, larvae (L1-L5), pupae (PP, Pw, Pp, Pb and Pd) and adults (A1, A10 and A30). Each value is presented as the mean (SD) of three replicates. Vertical bars represent the mean \pm SEM $(n=3)$. Letters above the bars indicate significant differences $(P<0.001)$ identified by SAS software version 9.1 


\subsection{Developmental expression pattern of $A c c A I F 3$}

Total RNA from the larvae, pupae and adults was extracted to investigate the expression pattern of AccAIF3 at different developmental stages using qRT-PCR. In Figure 5, the results indicate that AcCAIF3 is rarely expressed in eggs. High expression levels of AccAIF3 were detected in the $\mathrm{Pd}, \mathrm{A} 1$ and $\mathrm{A} 30$ stages, and the expression of AccAIF3 peaked at the A15 stage.

\subsection{The expression profile of AccAIF3 under environmental stresses, and exposure to hormones and fungus}

To characterise the influence of temperature on the AccAIF3 transcriptional level, qRT-PCR was performed. The expression levels were normalised to those of control worker honeybees. Under cold $\left(4{ }^{\circ} \mathrm{C}\right)$ treatments, the expression of AccAIF3 reached a peak after $1.0 \mathrm{~h}$, followed by a sharp decrease (Fig. 6a), while heat treatment $\left(42^{\circ} \mathrm{C}\right)$
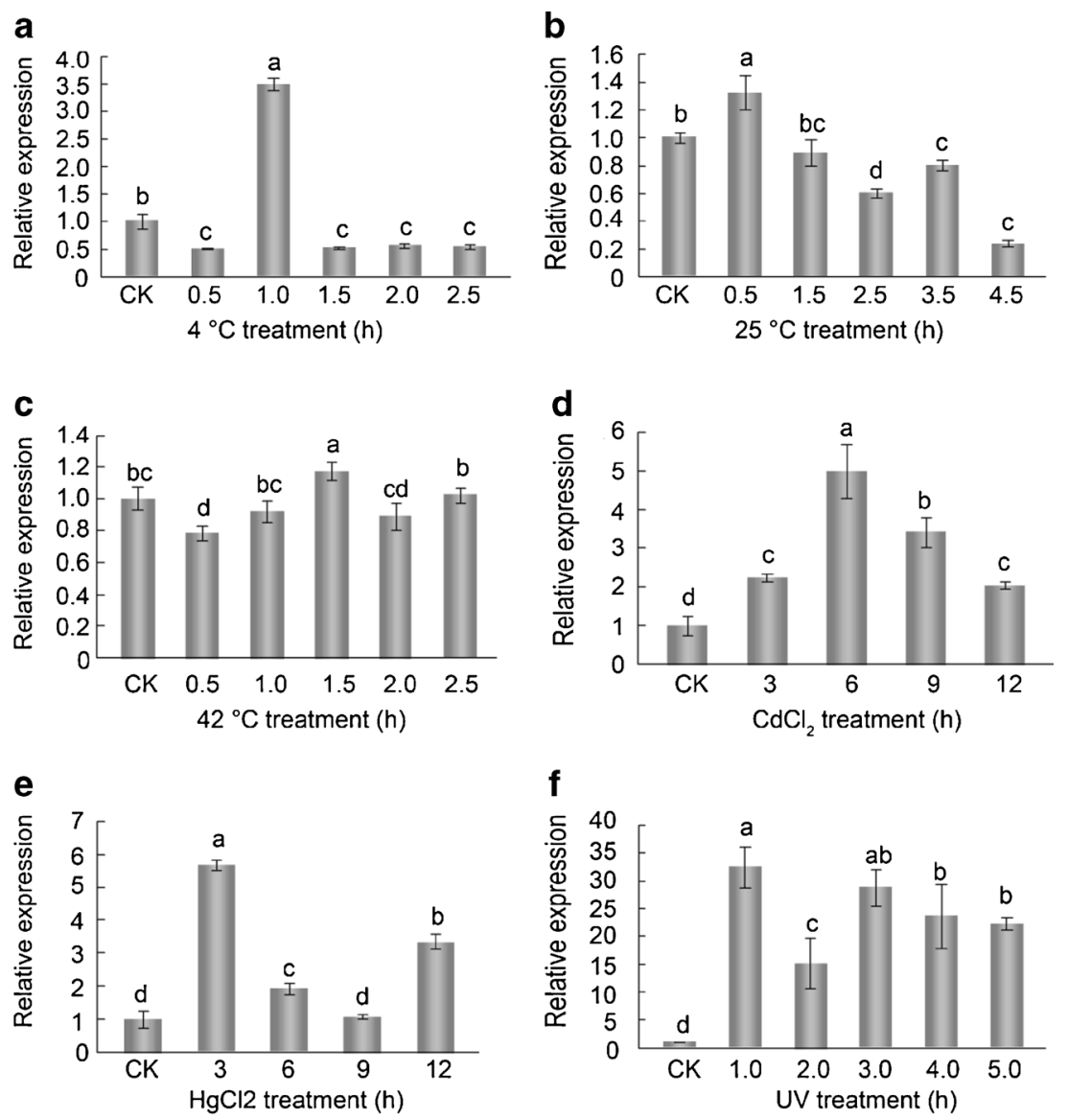

Figure 6 The expression profiles of AccAIF3. a-c The expression of AccAIF3 under various temperature stress treatments, with the $\beta$-actin gene shown for comparison at the appropriate time. $\mathbf{d}$ and $\mathbf{e}$ The transcription level of AccAIF3 under heavy metal treatment with $\mathrm{CdCl}_{2}$ and $\mathrm{HgCl}_{2}$. f $A c c A I F 3 \mathrm{mRNA}$ expression level after exposure to UV treatment at the appropriate time. CK is the abbreviation for control check. Vertical bars represent the mean \pm SEM $(n=3)$. Letters above the bars indicate significant differences $(P<0.001)$ identified by SAS software version 9.1 . 
had slight significant influence on the AccAIF3 transcriptional level (Fig. 6c). Under $25{ }^{\circ} \mathrm{C}$ treatment, the level of AccAIF3 transcription was induced within $0.5 \mathrm{~h}$ (Fig. 6b).

Under $\mathrm{CdCl}_{2}$ treatment, the transcriptional rates of $A c c A I F 3$ were induced with a peak at $6 \mathrm{~h}$ (Fig. 6d). Different from $\mathrm{CdCl}_{2}$ treatment, $\mathrm{HgCl}_{2}$ treatment caused a nearly 6.0 -fold relative expression increase in AccAIF3 (Fig. 6e). In addition, UV treatment caused a drastic upregulation of AccAIF3 expression (Fig. 6f), which indicated that AccAIF3 might be associated with UV-related activation.
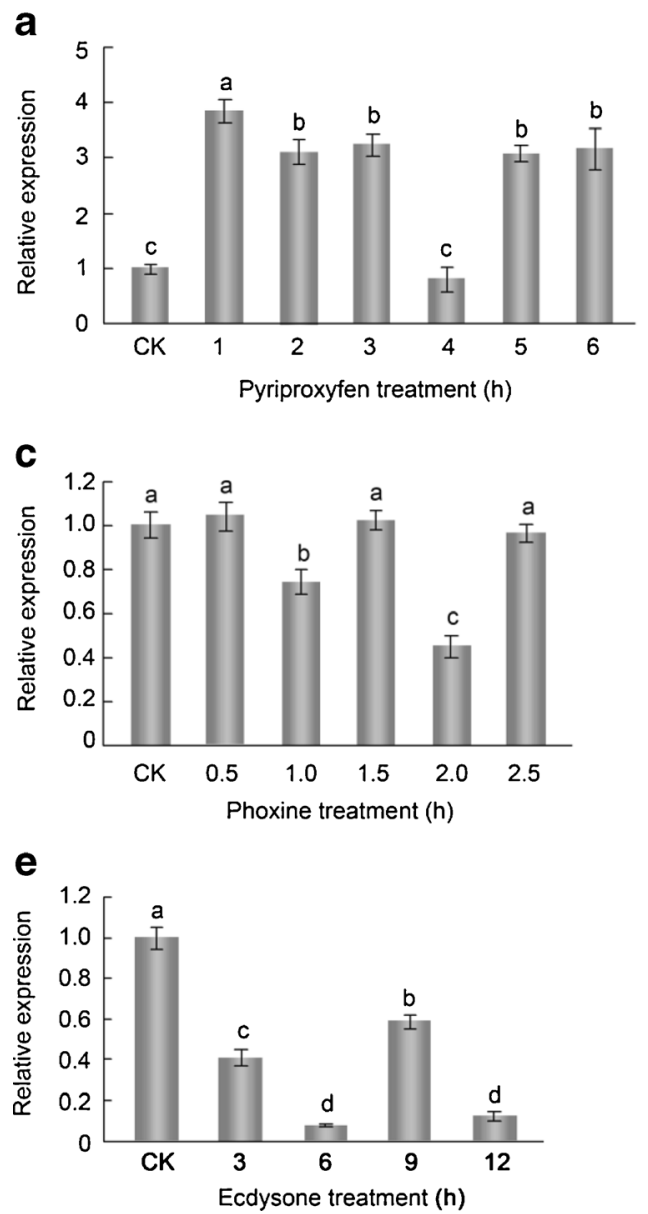

As worker honeybees work within the environment, they contact unavoidable pesticides, which may lead to death. To study the potential role of AccAIF3 against pesticides, qRT-PCR was performed. Treatment with pyriproxyfen persistently induced a sharply increase in the level of AccAIF3 transcription, except at $4 \mathrm{~h}$ (Fig. 7a). The transcript level of AccAIF3 was highest at $2 \mathrm{~h}$ following cyhalothrin treatment (Fig. 7b). In contrast to pyriproxyfen and cyhalothrin, no significant alteration was observed after phoxime treatment (Fig. 7c). To study the response to ecdysone, which is a

b
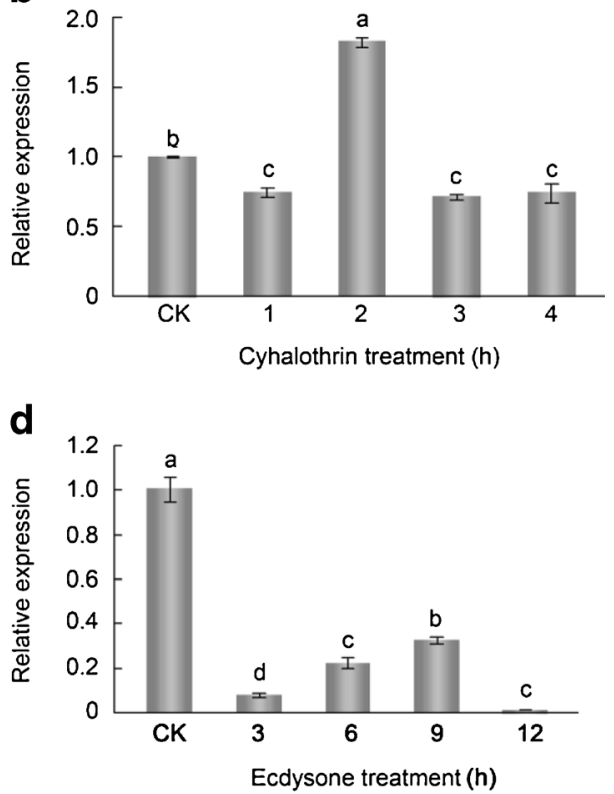

Figure 7 The transcript level of AccAIF3 under pesticide stress. a-c The expression level of AccAIF3 under pyriproxyfen, cyhalothrin and phoxime treatment. The ecdyson treatments were $1.0 \mu \mathrm{g} / \mathrm{mL}$ (d) and $0.001 \mu \mathrm{g} /$ $\mathrm{mL}$ (e). CK is the abbreviation for control check. Each value is presented as the mean (SD) of three replicates. Letters above the bars indicate significant differences $(P<0.001)$ identified by SAS software version 9.1 
hormone that influences the development of larvae to pupae, $1.0 \mu \mathrm{g} / \mathrm{mL}$ and $0.001 \mu \mathrm{g} / \mathrm{mL}$ of ecdysone were used to treat larvae separately. Figure $7 \mathrm{~d}$ and e showed that the transcriptions of AccAIF3 were noticeably inhabited.

Ascosphaera apis, a primary fungus, threatens the life of the honeybee. To detect the differences in AccAIF3 and AmAIF3 in response to Ascosphaera apis, qRT-PCR was performed. Figure 8 showed that the transcript rate of AcCAIF3 reached a peak at approximately 5.0 -fold relative to the normal, while that of AIF3 in A. mellifera L. was not significantly altered, which showed that AccAIF3 might play an important role in resistance to Ascosphaera apis.

\section{DISCUSSION}

Apoptosis is pivotal for the development and maintenance of healthy tissues by removing damaged and mutated cells and preventing the induction of cancer. The deregulation of apoptosis leads to the progression of tumours and malignancy due to an accumulation of gene mutations and genetic instability (Johnstone et al. 2002; Kitada et al. 2002). AIFs are factors

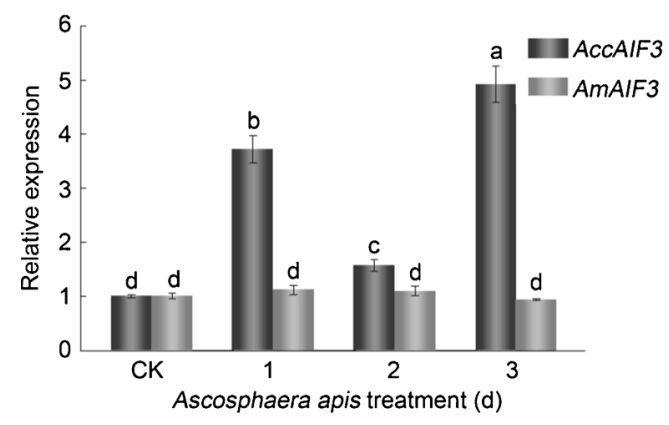

Figure 8 Functional comparison of AccAIF3 and AmAIF3 after exposure Ascosphaera apis for $1 \mathrm{~d}, 2$ $\mathrm{d}$ and $3 \mathrm{~d}$. CK is the abbreviation for control check. The expression levels of AccAIF3 are indicated with grey boxes, and the transcription of AmAIF3 is indicated with black boxes. Vertical bars represent the mean \pm SEM $(n=3)$. Letters above the bars indicate significant differences $(P<0.001)$ identified by SAS software version 9.1 involved in apoptosis. Although many studies have focused on the mammals AIFs, few reports have investigated AIFs in insects. In this study, we reported the first cloning and characterisation of the AIF3 gene from A. cerana cerana, and we have studied its expression level in response to various stresses.

AmAIF3 has another name in NCBI: $A$. mellifera thioredoxin reductase 3 (AmTrxR3) (GenBank accession number: XM_625032.4). TrxRs is a flavoprotein that contains an FAD prosthetic group, an NADPH binding site and an active site (Mustacich and Powis 2000). Studies have demonstrated that TrxRs in their oxidised form can protect organisms against oxidant injury (Yang et al. 2010a). In addition, TrxRs also play roles in cell growth and transformation, and the recycling of ascorbate (Mustacich and Powis 2000). Among all known enzymatic functions of eukaryotic proteins, AIF has the strongest homology with plant semidehydroascorbate and ascorbate reductases (Susin et al. 1999). In this respect, AccAIF3 may have a potential function such as AmTrxR3 in maintaining the redox homeostasis of a cell.

To better understand the potential roles of AccAIF3, the 5' flanking region was isolated. Cell factor 2-II (CF2-II), related to development and growth (Stanojević et al. 1989), was identified. Therefore, we studied the expression pattern of AccAIF3 throughout development. Adult worker honeybees have to forage for pollen and nectar at 2-3 weeks of age (Ament et al. 2008). We hypothesised that when worker honeybees fly to forage, the expression of AccAIF3 is needed to protect organisms against various environmental stresses. Interestingly, the qRT-PCR results demonstrated that the transcript level of AccAIF3 was highest at A15 (Fig. 5). This stage is approximately when worker honeybees begin to forage outside.

Other TFBS heat shock factors (HSFs) related to heat-induced transcriptional activation (Fernandes et al. 1994) were also found in the region. Temperature can cause physiological changes in organisms and is one of the most important abiotic environmental factors (An and 
Choi 2010). To avoid harm caused by temperature, organisms have evolved many strategies, such as changing the cell membrane fluidity, expressing some proteins and seeking shelter (Yang et al. 2010b). To detect whether AccAIF3 was associated with temperature stress, the expression levels of AccAIF3 were measured. The data showed that the AccAIF3 transcription levels were significantly increased in a short time when bees exposed to $4{ }^{\circ} \mathrm{C}$ and $25{ }^{\circ} \mathrm{C}$, while no significant alteration was observed at $42{ }^{\circ} \mathrm{C}$ (Fig. 6a-c), indicating that A. cerana cerana may adapt to heat conditions. These data indicated that AcCAIF3 might be associated with temperature-induced transcriptional activation. In addition, binding sites for Broad-Complex (BR-C), which contributes to the tissue-specific response to ecdysone (von Kalm et al. 1994), were identified in the promoter. To study the responses of AccAIF3 to ecdysone, qRT-PCR was performed. Results indicated that the transcription of AccAIF3 was inhibited in response to different concentrations of ecdysone (Fig. 7d-e). The reasons that the results were opposite to the function of the putative BR-C may be due to the material, the treatment method and the collection time.

In addition to the TFBS mentioned above, CdxAs were also identified. Frumkin et al. (1994) demonstrated that CdxAs play roles in gut closure, indicating that AccAIF3 may be involved in gut protection. Some diseases initially occur in the gut. For example, Ascosphaera apis is a filamentous fungus that causes Chalkbrood (Williams 2000; Hornitzky 2001). After ingestion, the fungal spores germinate in the larval gut, and then mycelia cross the gut lining and proliferate through the body cavity. Chalkbrood can lead to heavy losses in affected honeybee colonies (Cornman et al. 2012). At present, very few studies have focused on the molecular mechanism of honeybee immune responses to Chalkbrood (Aronstein and Saldivar 2005). In this study, a comparison was performed to investigate the changes in AccAIF3 and AmAIF3 after exposure to Ascosphaera apis. Our data indicated that despite the high homology between AccAIF3 and $A m A I F 3$, the responses were different (Fig. 8). AccAIF3 may be involved in the protection against Ascosphaera apis, while AmAIF3 may not be relevant. This phenomenon perhaps resulted from the cis-acting factors Dfd, which determine the specificity of homeotic gene action (Ekker et al. 1992).

Furthermore, we also detected changes in the expression levels of AccAIF3 with exposure to various environmental stresses, such as heavy metals, UV, and pesticides. Heavy metal contamination remains a critical environmental problem (Nagajyoti et al. 2010). Worker honeybees foraging for nectar and pollen outside may contact heavy metals. qRT-PCR results revealed that both $\mathrm{CdCl}_{2}$ and $\mathrm{HgCl}_{2}$ induce the transcript level of AccAIF3 in a time-dependent manner (Fig. 6d-e), and AccAIF3 may function in avoiding injury. UV is harmful to organisms and can destroy the structure of DNA, inhibit DNA repair pathways and interfere with the functions of proteins (Park et al. 2000). The transcript levels of AccAIF3 were dramatically enhanced by UV treatment (Fig. 6f), suggesting a role for AcCAIF3 in the enzymatic protection against oxidative stress. The excessive use of pesticides has a negative impact on the normal development of the digestive system and glands in insects (Franco et al. 2009). Figure 7a-c showed that AccAIF3 might protect bees against pesticides. Murakami and Johnson (1996) revealed that increased resistance to environmental stresses is necessary for protection, and enhanced resistance to ROS may be efficient for the extension of life in the fruit fly.

In conclusion, we cloned the AIF3 gene from A. cerana cerana, and the expression of AccAIF3 peaked at the A15 stage during development. The transcript levels of AccAIF3 were significantly increased by exposure to cold, $\mathrm{HgCl}_{2}, \mathrm{CdCl}_{2}, \mathrm{UV}$, pesticides and Ascosphaera apis. The increased expression level perhaps partially corresponds to the increased levels of ROS. Taken together, AccAIF3 may have an important influence on the response to environmental stresses and result in the elevated resistance of honeybees to adversities. 


\section{ACKNOWLEDGMENTS}

This work was financially supported by the earmarked fund for the China Agriculture Research System (No.CARS-45), Agro-scientific Research in the Public Interest (No. 200903006), and the National Natural Science Foundation (No. 31172275) in China.

Identification moléculaire et réponse au stress du gène 3 codant pour le facteur inducteur d'apoptose (AccAIF3) chez Apis cerana cerana

Stress biotique / stress abiotique / PCR en temps réel / oxydo-réductase / Ascosphaera apis

Molekulare Charakterisierung und Stressantwort des Apoptose-induzierenden Faktor Gens 3 (AccAIF3) von Apis cerana cerana

Apis cerana cerana / Biotischer and abiotischer Stress / Quantitative Real-Time PCR / AccAIF3

\section{REFERENCES}

Alaux, C., Ducloz, F., Crauser, D., Le Conte, Y. (2010) Diet effects on honeybee immunocompetence. Biol. Lett. 6, 562-565

Ament, S.A., Corona, M., Pollock, H.S., Robinson, G.E. (2008) Insulin signaling is involved in the regulation of worker division of labor in honey bee colonies. Proc. Natl. Acad. Sci. U. S. A. 105, 4226-4231

An, M.I., Choi, C.Y. (2010) Activity of antioxidant enzymes and physiological responses in ark shell, Scapharca broughtonii, exposed to thermal and osmotic stress: Effects on hemolymph and biochemical parameters. Comp. Biochem. Physiol. Biochem. Mol. Biol. 155(1), 34-42

Apostolova, N., Cervera, A.M., Victor, V.M., Cadenas, S., Sanjuan-Pla, A., Alvarez-Barrientos, A., Esplugues, J.V., McCreath, K.J. (2006) Loss of apoptosis-inducing factor leads to an increase in reactive oxygen species, and an impairment of respiration that can be reversed by antioxidants. Cell Death Differ. 13, 354-357

Aronstein, K., Saldivar, E. (2005) Characterization of a honey bee Toll related receptor gene Am18w and its potential involvement in antimicrobial immune defense. Apidologie 36(1), 3-14

Cornman, R.S., Bennett, A.K., Murray, K.D., Evans, J.D., Elsik, C.G., Aronstein, K. (2012) Transcriptome analysis of the honey bee fungal pathogen, Ascosphaera apis: implications for host pathogenesis. BMC Genomics 13(1), 285
Delettre, C., Yuste, V.J., Moubarak, R.S., Bras, M., Lesbordes-Brion, J.C., Petres, S., Bellalour, J., Susin, S.A. (2006a) AIFsh, a novel apoptosisinducing factor (AIF) pro-apoptotic isoform with potential pathological relevance in human cancer. J. Biol. Chem. 281, 6413-6427

Delettre, C., Yuste, V.J., Moubarak, R.S., Bras, M., Robert, N., Susin, S.A. (2006b) Identification and characterization of AIFsh2, a mitochondrial apoptosis-inducing factor (AIF) isoform with NADH oxidase activity. J. Biol. Chem. 281, 18507-18518

Ekker, S.C., von Kessler, D.P., Beachy, P.A. (1992) Differential DNA sequence recognition is a determinant of specificity in homeotic gene action. EMBO J. 11(11), 4059

Fernandes, M., Xiao, H., Lis, J.T. (1994) Fine structure analyses of the Drosophila and Saccharomyces heat shock factor-heat shock element interactions. Nucleic Acids Res. 22(2), 167-173

Franco, R., Sánchez-Olea, R., Reyes-Reyes, E.M., Panayiotidis, M.I. (2009) Environmental toxicity, oxidative stress and apoptosis: menage a trois. Mutat. Res. 674(1), 3-22

Frumkin, A., Pillemer, G., Haffner, R., Tarcic, N., Gruenbaum, Y., Fainsod, A. (1994) A role for CdxA in gut closure and intestinal epithelia differentiation. Development 120(2), 253-263

Garrido, C., Schmitt, E., Cande, C., Vahsen, N., Parcellier, A., Kroemer, G. (2003) HSP27 and HSP70: potentially oncogenic apoptosis inhibitors. Cell Cycle 2, 579-584

Green, D.R., Reed, J.C. (1998) Mitochondria and apoptosis. Science 281, 1309-1312

Hangen, E., Kroemer, G., Modjtahedi, N. (2009) Vital functions of Apoptosis Inducing Factor (AIF). Gastroenterol Hepatol Bed Bench. 2009, 2(1)

Hangen, E., Blomgren, K., Bénit, P., Kroemer, G., Modjtahedi, N. (2010) Life with or without AIF. Trends Biochem. Sci. 35(5), 278-287

Hornitzky, M. (2001) Literature review of chalkbrood, a fungal disease of honeybees. Rural Industries Research and Development Corporation. RIRDC Publ. 1, 150

Johnstone, R.W., Ruefli, A.A., Lowe, S.W. (2002) Apoptosis: a link between cancer genetics and chemotherapy. Cell 108(2), 153-164

Joza, N., Susin, S.A., Daugas, E., Stanford, W.L., Cho, S.K., et al. (2001) Essential role of the mitochondrial apoptosis-inducing factor in programmed cell death. Nature 410(6828), 549-554

Joza, N., Galindo, K., Pospisilik, J.A., Benit, P., Rangachari, M., et al. (2008) The molecular archaeology of a mitochondrial death effector: AIF in Drosophila. Cell Death Differ. 15(6), 1009-1018

Kitada, S., Pedersen, I.M., Schimmer, A.D., Reed, J.C. (2002) Dysregulation of apoptosis genes in hematopoietic malignancies. Oncogene 21(21), 3459-3474 
Klein, J.A., Longo-Guess, C.M., Rossmann, M.P., Seburn, K.L., Hurd, R.E., Frankel, W.N., Bronson, R.T., Ackerman, S.L. (2002) The harlequin mouse mutation downregulates apoptosis-inducing factor. Nature 419(6905), 367-374

Kroemer, G. (1997) The proto-oncogene Bcl-2 and its role in regulating apoptosis. Nat. Med. 3(6), 614620

Kroemer, G., Reed, J.C. (2000) Mitochondrial control of cell death. Nat. Med. 6(5), 513-519

Li, H.L., Zhang, Y.L., Gao, Q.K., Cheng, J.A., Lou, B.G. (2008) Molecular identification of cDNA, immunolocalization, and expression of a putative odorantbinding protein from an Asian honey bee, Apis cerana cerana. J. Chem. Ecol. 34(12), 1593-1601

Loeffler, M., Daugas, E., Susin, S.A., Zamzami, N., Metivier, D., Nieminen, A.L., Borthers, G., Penninger, J.M., Kroemer, G. (2001) Dominant cell death induction by extramitochondrially targeted apoptosis-inducing factor. FASEB J. 15, $758-767$

Lorenzo, H.K., Susin, S.A., Penninger, J., Kroemer, G. (1999) Apoptosis inducing factor (AIF): a phylogenetically old, caspase-independent effector of cell death. Cell Death Differ. 6(6), 516

Maté, M.J., Ortiz-Lombardía, M., Boitel, B., Haouz, A., Tello, D., Susin, S.A., Penninger, J., Kroemer, G., Alzari, P.M. (2002) The crystal structure of the mouse apoptosis-inducing factor AIF. Nat. Struct. Mol. Biol. 9(6), 442-446

Michelette, E.R., Soares, A.E.E. (1993) Characterization of preimaginal developmental stages in Africanized honey bee workers (Apis mellifera L). Apidologie 24(4), 431-440

Miramar, M.D., Costantini, P., Ravagnan, L., Saraiva, L.M., Haouzi, D., Brothers, G., Penninger, J.M., Peleato, M.L., Kroemer, G., Susin, S.A. (2001) NADH oxidase activity of mitochondrial apoptosisinducing factor. J. Biol. Chem. 276(19), 1639116398

Mosser, D.D., Morimoto, R.I. (2004) Molecular chaperones and the stress of oncogenesis. Oncogene 23, 2907-2918

Murakami, S., Johnson, T.E. (1996) A genetic pathway conferring life extension and resistance to UV stress in Caenorhabditis elegans. Genetics 143, 1207-1218

Mustacich, D., Powis, G. (2000) Thioredoxin reductase. Biochem. J. 346, 1-8

Nagajyoti, P.C., Lee, K.D., Sreekanth, T.V.M. (2010) Heavy metals, occurrence and toxicity for plants: a review. Environ. Chem. Lett. 8(3), 199-216

Norberg, E., Orrenius, S., Zhivotovsky, B. (2010) Mitochondrial regulation of cell death: processing of apoptosis-inducing factor (AIF). Biochem. Biophys. Res. Commun. 396(1), 95-100

Ohiro, Y., Garkavtsev, I., Kobayashi, S., Sreekumar, K.R., Nantz, R., et al. (2002) A novel p53-inducible apoptogenic gene, PRG3, encodes a homologue of the apoptosis-inducing factor (AIF). FEBS Lett. 524(1), 163-171

Otera, H., Ohsakaya, S., Nagaura, Z.I., Ishihara, N., Mihara, K. (2005) Export of mitochondrial AIF in response to proapoptotic stimuli depends on processing at the intermembrane space. EMBO J. 24, 1375-1386

Park, S.H., Chung, Y.M., Lee, Y.S., Kim, H.J., Kim, J.S., Chae, H.Z., Yoo, Y.D. (2000) Antisense of human peroxiredoxin II enhances radiation-induced cell death. Clin. Cancer Res. 6(12), 4915-4920

Stanojević, D., Hoey, T., Levine, M. (1989) Sequencespecific DNA-binding activities of the gap proteins encoded by hunchback and Krüppel in Drosophila. Nature 341, 331-335

Susin, S.A., Lorenzo, H.K., Zamzami, N., Marzo, I., Snow, B.E., et al. (1999) Molecular characterization of mitochondrial apoptosis-inducing factor. Nature 397(6718), 441-446

Thompson, C.B. (1995) Apoptosis in the pathogenesis and treatment of disease. Science 267(5203), 1456-1462

Urbano, A., Lakshmanan, U., Choo, P.H., Kwan, J.C., Ng, P.Y., Guo, K., Dhakshinamoorthy, S., Porter, A. (2005) AIF suppresses chemical stress-induced apoptosis and maintains the transformed state of tumor cells. EMBO J. 24(15), 2815-2826

von Kalm, L., Crossgrove, K., Von Seggern, D., Guild, G.M., Beckendorf, S.K. (1994) The Broad-Complex directly controls a tissue-specific response to the steroid hormone ecdysone at the onset of Drosophila metamorphosis. EMBO J. 13(15), 3505

Williams, D.L. (2000) A veterinary approach to the European honey bee (Apis mellifera). Vet. J. 160, 61-67

Wu, M., Xu, L.G., Li, X., Zhai, Z., Shu, H.B. (2002) AMID, an apoptosis-inducing factor-homologous mitochondrion-associated protein, induces caspaseindependent apoptosis. J. Biol. Chem. 277(28), 25617-25623

Xie, Q., Lin, T., Zhang, Y., Zheng, J., Bonanno, J.A. (2005) Molecular cloning and characterization of a human AIF-like gene with ability to induce apoptosis. J. Biol. Chem. 280(20), 19673-19681

Yan, H., Meng, F., Jia, H., Guo, X., Xu, B. (2012) The identification and oxidative stress response of a zeta class glutathione S-transferase (GSTZ1) gene from Apis cerana cerana. J. Insect Physiol. 58(6), 782791

Yang, H., Kang, M., Guo, X., Xu, B. (2010a) Cloning, structural features, and expression analysis of the gene encoding thioredoxin reductase 1 from Apis cerana cerana. Comp. Biochem. Physiol. Biochem. Mol. Biol. 156(3), 229-236

Yang, L.H., Huang, H., Wang, J.J. (2010b) Antioxidant responses of citrus red mite, Panonychus citri (McGregor) (Acari: Tetranychidae), exposed to thermal stress. J. Insect Physiol. 56, 1871-1876 
Yao, P., Hao, L., Wang, F., Chen, X., Yan, Y., et al. (2013) Molecular cloning, expression and antioxidant characterisation of a typical thioredoxin gene (AccTrx2) in Apis cerana cerana. Gene 527(1), 33-41

Yao, P., Chen, X., Yan, Y., Liu, F., Zhang, Y., Guo, X., Xu, B. (2014) Glutaredoxin 1, glutaredoxin 2 , thioredoxin 1 , and thioredoxin peroxidase 3 play important roles in antioxidant defense in
Apis cerana cerana. Free Radic. Biol. Med. 68, 335-346

Ye, H., Cande, C., Stephanou, N.C., Jiang, S., Gurbuxani, S., Larochette, N., Daugas, E., Garrido, C., Kroemer, G., Wu, H. (2002) DNA binding is required for the apoptogenic action of apoptosis inducing factor. Nat. Struct. Mol. Biol. 9(9), 680684 